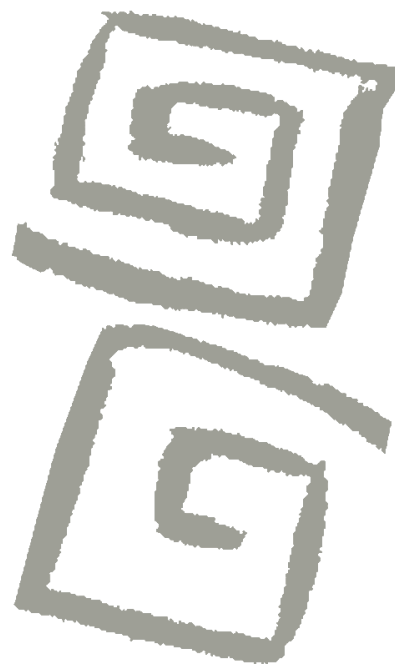

\title{
Muertes por accidente de motocicleta y su asociación con variables relacionadas a la reproducción social en un estado del noreste brasileño
}

\author{
Deaths due to motorcycle accidents and their \\ association with variables related to social \\ reproduction in a northeastern Brazilian state
}

Silva, Paul Hindenburg Nobre de Vasconcelos ${ }^{1}$; Lima, Maria Luiza Carvalho²; Souza, Wayner Vieira ${ }^{3}$; Moreira, Rafael da Silveira"; Oliveira, Fernando José Moreira $^{5}$

'Doctor en Salud Pública, Analista de Gestión en Salud. Centro de Pesquisas Aggeu Magalhães, Fundação Oswaldo Cruz. Recife, Brasil. paulnobre@cpqam.fiocruz.br

${ }^{2}$ Doctora en Salud Pública. Investigadora, Departamento de Saúde Coletiva, Centro de Pesquisas Aggeu Magalhães, Fundação Oswaldo Cruz. Recife, Brasil.

luiza@cpqam.fiocruz.br

${ }^{3}$ Doctor en Salud Pública. Investigador, Departamento de Saúde Coletiva, Centro de Pesquisas Aggeu Magalhães, Fundação Oswaldo Cruz. Recife, Brasil.

wayner@cpqam.fiocruz.br

${ }^{4}$ Doctor en Salud Pública. Investigador, Departamento de Saúde Coletiva, Centro de Pesquisas Aggeu Magalhães, Fundação Oswaldo Cruz. Recife, Brasil.

moreirars@cpqam.fiocruz.br

${ }^{5}$ Magíster en Salud Pública. Gerente de Información en Salud. Secretaria de Saúde do Estado de Pernambuco. Recife, Brasil.

fermando_moreira35@ hotmail.com
RESUMEN El objetivo de este artículo es identificar el comportamiento de las muertes por accidentes de motocicleta y las variables relacionadas con la teoría de la reproducción social de Samaja, para el período 2000-2005 en el estado de Pernambuco, Brasil. Se realizó un estudio ecológico con abordaje caso-control. La unidad de análisis fue el municipio. Los casos fueron definidos considerando el $20 \%$ de los municipios con los mayores coeficientes bayesianos empíricos locales de mortalidad por accidentes de motocicleta y los controles, como el $40 \%$ de los municipios con menores coeficientes de mortalidad por accidentes de motocicleta. Los municipios con mayor probabilidad de altos coeficientes de mortalidad por accidentes de motocicleta mostraron factores de crecimiento poblacional altos, así como de crecimiento de la flota de vehículos, bajas densidades demográficas, bajo PBI per cápita, y más de 20 motocicletas por mil habitantes. Se concluye que las variables relacionadas a las macropolíticas mostraron una mayor fuerza para explicar las probabilidades de defunciones por accidentes de motocicleta.

PALABRAS CLAVES Accidentes de Tránsito; Mortalidad; Motocicletas; Estudios de Casos y Controles; Estudios Ecológicos; Brasil.

ABSTRACT The objective of this article was to identify the association between motorcycle deaths and variables related to Samaja's theory of social reproduction in the period 2000-2005 in the state of Pernambuco. An ecological, case-control study was carried out, with municipalities as the unit of analysis. Cases were defined as the $20 \%$ of municipalities with the highest local empirical Bayesian coefficients for mortality due to motorcycle accidents, and controls as the $40 \%$ with the lowest coefficients. The municipalities with the greatest chances of high coefficients for mortality due to motorcycle accidents showed high population growth factors and increases in the total fleet of motorcycles, with low population densities, low GDP per capita, and more than 20 motorcycles per thousand inhabitants. We conclude that the variables related to macropolicies proved to have greater force in explaining higher chances of motorcycle death. KEY WORDS Accidents, Traffic; Mortality; Motorcycles; Case-Control Studies; Ecological Studies; Brazil. 


\section{INTRODUCCIÓN}

Se estima que en Brasil cada 15 minutos muere una persona debido a accidentes de tránsito (1). Las consecuencias socioeconómicas representan un costo de alrededor de 16 billones de dólares estadounidenses anuales, a valor actualizado para abril de 2011 por el Instituto de Pesquisa Económica Aplicada (IPEA) $(2,3)$; además de la desestructuración de núcleos familiares, sufrimiento, secuelas y otras consecuencias que no son mensuradas, pero que tienen un alto impacto en la calidad de vida de las personas (4).

Los denominados países en desarrollo como Brasil representan cerca del $90 \%$ de las muertes en el mundo por accidentes de tránsito, en tanto que poseen apenas el $48 \%$ de la flota de vehículos automotores y tienen como víctimas principales a los peatones, ciclistas y motociclistas (5).

El padrón epidemiológico de los accidentes de tránsito en la realidad brasileña presenta una característica fundamental: ocurre con intensidad variada y distribución heterogénea por el territorio nacional, con fuerte tendencia creciente en muchos municipios, principalmente con poblaciones de pequeño (menores a 50.000 habitantes) y mediano porte (entre 50.000 a 100.000 habitantes). Sorprende el crecimiento de accidentes de motociclistas, con aumento de defunciones e incapacidad en proporciones mucho más elevadas que en otras modalidades de transporte (6).

En el estado de Pernambuco, la tasa de mortalidad de motociclistas creció el 875\% entre 1996 (0,4 por 100 mil habitantes) y 2006 (3,9 por 100 mil habitantes) (8). Aun así, todavía son escasos los estudios que buscan comprender y explicar estos fenómenos más allá de los paradigmas de la fragmentación y el reduccionismo positivista. Los programas para la promoción de la seguridad y prevención de los accidentes que existen en Brasil desde hace cuarenta años, siguieron ese camino. Ninguno contempla los conceptos de determinación y reproducción social en la comprensión y explicación de esos agravios a la salud.

En este estudio, para interpretar los resultados, se utiliza la teoría de la reproducción social de Samaja. Esta teoría se fundamenta en dos conceptos y un principio metodológico. El primer concepto considera la vida humana como una articulación compleja de múltiples procesos histórico-sociales de producción y reproducción de sí misma, a lo largo de la cual surgen tensiones y conflictos que motivan acciones de reparación y transformación. El segundo concepto enuncia que los resultados de los procesos histórico-sociales se configuran por medio de estratos, en diversos niveles de integración, ordenados como jerarquías estructurales y funcionales que se organizan en sentido ascendente, siguiendo el sentido de su producción y en sentido descendente siguiendo el sentido de la reproducción $(9,10)$.

El principio metodológico postula que es preciso investigar no solamente la composición y el funcionamiento de las estructuras (organismo, individuo, familia, mercado, etc.), sino también, y sobre todo, la historia: esto es, los procesos de surgimiento de algunos niveles a partir de los conflictos y desequilibrios anteriores $(9,10)$.

Para Milton Santos (11) y Samaja (12), el concepto de espacio trasciende la noción de superficie y área geográfica incorporando también los aspectos sociales; dado que se trata de una instancia de la sociedad, así como lo son la instancia económica, la político-institucional y la culturalideológica, se debe vincular la ocurrencia de estos eventos al espacio donde ocurren.

Esta categoría, al ser utilizada en este estudio por medio de la unidad de análisis municipios, pretende rescatar ese abordaje y se adecua al modelo teórico de Samaja $(9,11)$.

El presente estudio tiene por objetivo identificar la asociación existente entre las muertes por accidentes de motocicleta y las variables relacionadas a las dimensiones de los procesos de reproducción social que pueden estar explicando su distribución espacial de forma heterogénea.

\section{MÉTODOS}

El área de estudio fue el estado de Pernambuco que tiene 184 municipios -excluyendo el archipiélago de Fernando de Noronha- agrupados en doce regiones de desarrollo.

Se trata de un estudio ecológico con abordaje caso-control, que tiene como unidad de análisis el municipio. Es un diseño de estudio híbrido utilizado de forma innovadora, tratando de comprender las 
diferencias de las situaciones asociadas a los estados de salud entre las poblaciones.

Se analizaron las defunciones por accidentes de motocicleta ocurridos en el estado de Pernambuco, entre el 1 de enero de 2000 y el 31 de diciembre de 2005. La muestra se compuso de casos y controles en una proporción de un caso cada dos controles. Los casos fueron definidos como el $20 \%$ de los municipios con mayores coeficientes bayesianos empíricos locales de mortalidad por accidente de motocicleta y los controles, como el $40 \%$ de los municipios con los menores coeficientes ya referidos, lo que resultó en un total de 37 municipios como casos y 75 como controles. Justifica este procedimiento la elección de situaciones extremas, para las que las características de cada grupo fueron más destacadas. Se respeta también, el criterio de que los controles formen parte de la misma población en la que se originan los casos, además de la inclusión en el análisis de la proporción de dos controles para cada caso $(13,14)$.

Los coeficientes se calcularon tomando las defunciones por accidentes de motocicleta como numerador y la población como denominador.

Para corregir las fluctuaciones aleatorias en pequeñas poblaciones o números pequeños de ocurrencias fueron estimados de nuevo los coeficientes de mortalidad por medio del método bayesiano empírico local, a partir de la suposición de que las tasas de áreas vecinas son autocorrelacionadas. Se reestimaron las tasas de esas áreas a través de la media ponderada entre el valor medido y la tasa media de vecindad, con pesos inversamente proporcionales a la población de cada área $(15,16)$.

El criterio de exclusión utilizado para los municipios fue aquellos que tenían más de un 10\% de defunciones mal definidas.

Se incluyeron todos las defunciones incluidas bajo el código "Motociclista lesionado en accidente de transporte" (V20-29) de la Clasificación Internacional de Enfermedades $10^{\circ}$ revisión (CIE 10) registrados en el Sistema de Información sobre Mortalidad (SIM) de residentes en el estado de Pernambuco ocurridos entre 2000 y 2005.

Las variables explicativas se seleccionaron con base en la bibliografía (17-21) y agrupadas según las dimensiones utilizadas por Samaja. En ese abordaje, la teoría de la reproducción social se da en cuatro dimensiones: biocomunal; conciencia y conducta; económica; y ecológico-política.

La reproducción biocomunal se refiere al modo en que sus miembros se renuevan corporalmente $y$ en sus interrelaciones, construyendo el medio comunal donde se realizan como individuos.

La reproducción de la autoconciencia y la conducta, que está relacionada con la producción de la cultura, se da a través de redes simbólicas mediadas por el lenguaje y la elaboración y transmisión de experiencias de aprendizaje (creencias, capacidades de interiorizar a los otros en función de la autoridad y de diferenciarse como singularidad personal). La reproducción económica, que abarca los medios de vida y de trabajo, no tiene como objeto inmediato la producción del propio organismo, sino la producción e intercambio de los bienes en todas sus escalas, mediando las reproducciones biocomunal, de la autoconciencia y de la conducta, y la ecológica-política.

La reproducción ecológico-política se refiere a las relaciones sociales responsables de la reproducción del macroambiente social y las condiciones territoriales/ambientales, que incluyen a las otras tres dimensiones (biocomunal, conciencia y conducta, y económica) y que, de cierta forma, las subordinan. Abarca las relaciones materiales y jurídicas de las macropolíticas que constituyen el Estado y fijan su esencia.

Los factores de crecimiento de la flota de automóviles y motocicletas impactan fuertemente en el ecosistema. En Brasil, esos factores también son estimulados por medio de macropolíticas estatales, como se explica más adelante. Por eso, fueron considerados en la dimensión ecológico-política.

Las fuentes de datos de las variables independientes corresponden al Censo 2000 del Instituto Brasileño de Geografía y Estadística (IBGE), la Agencia Estatal de Planificación e Investigaciones de Pernambuco y el Atlas de Desarrollo Humano del Programa de las Naciones Unidas para el Desarrollo (PNUD), el Departamento Estatal de Tránsito de Pernambuco (DETRAN) y el Departamento Nacional de Tránsito de Brasil (DENATRAN).

Las variables consideradas en cada dimensión fueron:

- Dimensión biocomunal: las muertes por accidentes de motociclistas representadas por sus coeficientes de mortalidad. 
- Dimensión económica: índice de GINI; razón del ingreso del $20 \%$ de los individuos más ricos sobre el $40 \%$ de los individuos más pobres; PIB per cápita; índice de desarrollo humano municipal (IDHM); porcentaje de jefes/as de hogar con un ingreso menor a dos y medio salarios mínimos; porcentaje de ingreso del 20\% de los individuos más ricos, porcentaje de ingreso del $20 \%$ de los individuos más pobres, porcentaje de la población con menos de cinco años de estudio, porcentaje de la población con más de 12 años de estudio.

- Dimensión ecológico-política: población, factor de crecimiento de la población al año; densidad demográfica; factor de crecimiento de la flota de automóviles al año; factor de crecimiento de la flota de motocicletas al año; factor de crecimiento de la flota total por año; razón del número de motocicletas por habitante; razón del número de automóviles por habitante; razón del número total de vehículos automotores por habitante; tránsito municipalizado (incluye fiscalización, señalización, operaciones de seguridad vial y prevención de accidentes, según el artículo 24 del Código de Tránsito Brasileño (CTB).

Los autores decidieron trabajar con estas tres dimensiones, dado que no se realizaron entrevistas con abordaje cualitativo para la dimensión de autoconciencia y conducta.

Luego de la construcción del banco de datos se realizó el análisis descriptivo de la distribución de las variables de estudio. Las variables explicativas fueron recodificadas asumiendo el valor uno cuando el valor originalmente observado fue superior a la mediana del conjunto de municipios de la muestra y cero en situación contraria; excepto en el caso de la variable "razón del número de motocicletas por habitante", en la que se utilizó la media como punto de corte para aumentar la sensibilidad del criterio. Con la utilización de este criterio para punto de corte quedó facilitada la comparación de situaciones extremas (municipios con los más altos coeficientes de mortalidad por accidentes de motocicleta versus los municipios con los coeficientes más bajos).

Para verificar la asociación entre el evento y las variables explicativas se realizó análisis multivariado con cálculo de odds ratio (OR) bruto y ajustado respectivamente, como medida de efecto.
Se definieron los intervalos con un 95\% de confianza. Las variables que presentaron asociación con un nivel de significancia por debajo del $25 \%$ $(p<0,25)$ fueron introducidas en el modelo multivariado de regresión logística. El método de selección de las variables utilizado fue el backward stepwise, con verificación estadística de la significancia utilizando el test de razón de verosimilitud y criterios para permanencia de la variable en el modelo $p<0,10$.

Se utilizaron los programas Excel y el Statistical Package for Social Sciences (SPSS) versión 17.0, para el procesamiento y análisis de los datos. Este estudio formó parte de la tesis de doctorado Epidemiología de los accidentes de tránsito con un enfoque en la mortalidad de motociclistas en el estado de Pernambuco: una exacerbación de la violencia social. Dicho proyecto fue aprobado por el Comité de Ética en Investigación del Centro de Pesquisas Aggeu Magalhães (CPqAM) dependiente de la Fundação Oswaldo Cruz (FIOCRUZ).

\section{RESULTADOS}

El Cuadro 1 presenta los datos de mortalidad por accidentes de motocicleta en el período en

Cuadro 1. Número de defunciones y tasa media de mortalidad por accidentes de motocicleta en los municipios caso y control. Pernambuco, Brasil, 2000-2005.

\begin{tabular}{lcc}
\hline Variables & Casos & Controles \\
\hline Defunciones por año & & \\
2000 & 60 & 45 \\
2001 & 58 & 53 \\
2002 & 65 & 62 \\
2003 & 83 & 87 \\
2004 & 69 & 121 \\
2005 & 82 & 110 \\
Total de defunciones & 417 & 478 \\
& & \\
Número de municipios & 37 & 75 \\
Población de los & 1.197 .838 & 5.086 .547 \\
municipios & & \\
Tasa media anual de & 5,80 & 1,57 \\
mortalidad (por 100 mil & & \\
habitantes) & & \\
\hline
\end{tabular}

Fuente: Elaboración propia. 
estudio según la situación de los municipios. Se destaca que en los municipios "caso" la tasa de mortalidad por accidente de motocicleta es casi cuatro veces mayor que en el grupo control.

El Cuadro 2 presenta las variables que tuvieron asociación significativa $(p<0,25)$ con el evento en el análisis bivariado. Así, no fueron incluidos en el análisis multivariado las variables: índice de desarrollo humano municipal; porcentaje de ingreso del 20\% más pobre; porcentaje de la población con menos de cinco años de estudio; porcentaje de la población con más de 12 años de estudio; tamaño poblacional; factor de crecimiento de la flota de automóviles al año; factor de crecimiento de la flota de motocicletas al año; razón del número de automóviles por habitante;

Cuadro 2. Defunciones por accidente de motocicleta en los municipios caso y control y variables explicativas, odds ratio bruta e intervalo de confianza del 95\%. Pernambuco, Brasil, 2000-2005.

\begin{tabular}{|c|c|c|c|c|c|}
\hline Variables/categorías & Casos & Controles & OR bruta & IC $95 \%$ & Valor $p$ \\
\hline \multicolumn{6}{|c|}{ Factor de crecimiento de la población al año } \\
\hline$>1,01$ & 24 & 32 & \multirow{2}{*}{2,48} & \multirow{2}{*}{1,$098 ; 5,607$} & \multirow{2}{*}{0,04} \\
\hline$<1,01$ & 13 & 43 & & & \\
\hline \multicolumn{6}{|c|}{ Factor de crecimiento de la flota total por año } \\
\hline$>1,10$ & 24 & 32 & \multirow{2}{*}{2,48} & \multirow{2}{*}{1,$098 ; 5,607$} & \multirow{2}{*}{0,04} \\
\hline$<1,10$ & 13 & 43 & & & \\
\hline \multicolumn{6}{|l|}{ Tránsito municipalizado } \\
\hline Sí & 1 & 10 & \multirow{2}{*}{5,54} & \multirow{2}{*}{0,$681 ; 45,028$} & \multirow{2}{*}{0,09} \\
\hline No & 36 & 65 & & & \\
\hline \multicolumn{6}{|c|}{ Densidad demográfica hab./km² } \\
\hline$>96,69$ & 5 & 51 & \multirow{2}{*}{0,07} & \multirow{2}{*}{0,$025 ; 0,212$} & \multirow{2}{*}{0,00} \\
\hline$<96,69$ & 32 & 24 & & & \\
\hline \multicolumn{6}{|l|}{ PIB per cápita $(\mathrm{R} \$)$} \\
\hline$>2.247,61$ & 27 & 29 & \multirow{2}{*}{4,28} & \multirow{2}{*}{1,$809 ; 10,137$} & \multirow{2}{*}{0,00} \\
\hline$<2.247,61$ & 10 & 46 & & & \\
\hline \multicolumn{6}{|l|}{ Índice de GINI } \\
\hline$>0,58$ & 19 & 27 & \multirow{2}{*}{0,53} & \multirow{2}{*}{0,$240 ; 1,184$} & \multirow{2}{*}{0,15} \\
\hline$<0,58$ & 18 & 48 & & & \\
\hline \multicolumn{6}{|c|}{ Porcentaje de ingreso del $20 \%$ más rico } \\
\hline$>60,61$ & 22 & 34 & \multirow{2}{*}{1,77} & \multirow{2}{*}{0,$796 ; 3,930$} & \multirow{2}{*}{0,23} \\
\hline$<60,61$ & 15 & 41 & & & \\
\hline \multicolumn{6}{|c|}{$\begin{array}{l}\text { Razón de ingreso del } 20 \% \text { más rico sobre el } \\
40 \% \text { más pobre }\end{array}$} \\
\hline$>14,44$ & 22 & 34 & \multirow{2}{*}{1,77} & \multirow{2}{*}{0,$796 ; 3,930$} & 020 \\
\hline$<14,44$ & 15 & 41 & & & 0,23 \\
\hline $\begin{array}{l}\text { Porcentaje de jefes/as de ho } \\
\text { ingreso menor a } 2,5 \text { salario }\end{array}$ & & & & & \\
\hline$>18,16$ & 13 & 43 & 04 & 0170.0011 & 004 \\
\hline$<18,16$ & 24 & 32 & 0,4 & $0,1 / 8 ; 0,911$ & 0,04 \\
\hline $\begin{array}{l}\text { Razón del número de motoc } \\
\text { habitantes }\end{array}$ & & & & & \\
\hline$>20$ motos & 22 & 20 & 402 & 1755.9970 & 000 \\
\hline$<20$ motos & 15 & 55 & & & \\
\hline
\end{tabular}

Fuente: Elaboración propia. 
Cuadro 3. Defunciones por accidente de motocicleta y variables explicativas, odds ratio ajustados, intervalos de confianza del $95 \%$ y valor de $p$, en análisis multivariado. Pernambuco, Brasil, 2000-2005.

\begin{tabular}{|c|c|c|c|}
\hline Variable & OR ajustado & IC $95 \%$ & Valor $p$ \\
\hline \multicolumn{4}{|c|}{ Factor de crecimiento de la población al año } \\
\hline$<1,01$ & 1 & - & - \\
\hline$>1,01$ & 5,53 & 1,$65 ; 18,54$ & 0,01 \\
\hline \multicolumn{4}{|c|}{ Factor de crecimiento de la flota total por año } \\
\hline$<1,10$ & 1 & - & - \\
\hline$>1,10$ & 2,60 & 0,$85 ; 7,95$ & 0,09 \\
\hline \multicolumn{4}{|c|}{ Densidad demográfica hab./km² } \\
\hline$<96,69$ & 1 & - & - \\
\hline$>96,69$ & 0,05 & 0,$01 ; 0,22$ & 0,00 \\
\hline \multicolumn{4}{|c|}{ PIB per cápita $(\mathrm{R} \$)$} \\
\hline$<2.247,61$ & 1 & - & - \\
\hline$>2.247,61$ & 3,62 & 1,$05 ; 12,45$ & 0,04 \\
\hline \multicolumn{4}{|c|}{ Razón del número de motocicletas/1.000 habitantes } \\
\hline$<20$ motos & 1 & - & - \\
\hline$>20$ motos & 9,22 & 2,$68 ; 31,65$ & 0,00 \\
\hline
\end{tabular}

Fuente: Elaboración propia.

razón del número total de vehículos automotores por habitante. Las variables que resultaron con una asociación significativa con la variable dependiente en el análisis multivariado están presentadas en el Cuadro 3.

Nótese que el modelo final obtenido muestra el perfil de los municipios con exceso de exposición y que estos tienen más chances de presentar mayores tasas de mortalidad por accidentes de motocicleta. Este perfil caracteriza como de riesgo mayor a los municipios con altos factores de crecimiento poblacional, altos factores de crecimiento de la flota de vehículos total, bajas densidades demográficas, bajo PIB per cápita, más de 20 motocicletas por 1.000 habitantes.

\section{DISCUSIÓN}

El hallazgo más importante del estudio fue mostrar empíricamente la relación de crecimiento acelerado de la población, el aumento de la flota de vehículos y la razón moto/habitante con las muertes por accidentes de tránsito, dato que no siempre es considerado cuando se aborda la temática. De esta forma se da continuidad a un tema abordado en la investigación "Estudio espacial de la mortalidad por accidentes de motocicleta en Pernambuco" (8), cuyos resultados sugieren la necesidad de buscar las asociaciones de esas muertes con las variables que mejor lo expliquen. Por esto fue utilizado el mismo período temporal y el banco de datos de la investigación citada. Además se verifica que la situación permanece prácticamente inalterada, por lo que no se requiere de la elaboración de otro estudio.

Las variables factor de crecimiento de la flota de automóviles y factor de crecimiento de la flota de motocicletas, consideradas en la dimensión ecológico-política se basan en que la fabricación cada vez mayor de automóviles y motocicletas y la idea de la motorización de la sociedad como "progreso", representada incluso como el acceso de los pobres a vehículos, configuran macropolíticas estatales que se propagan en otras dimensiones y de cierta forma las subordinan.

A partir de un estudio ecológico con abordaje caso-control -una alternativa a otras formas de análisis tradicionalmente utilizadas- que aplica 
el modelo explicativo de la reproducción social de Samaja, se incorporan referenciales de la determinación social para la interpretación de los resultados. Así, a través de un abordaje creativo se mostró que las variables con asociaciones estadísticamente significantes con las defunciones por accidentes de motocicleta son aquellas agrupadas en la dimensión ecológico-política, dimensión que está determinada por las políticas hegemónicas del aparato estatal y que se reproducen imbricándose en las otras dimensiones de forma descendente y ascendente.

En la presente discusión, se muestra cómo la historia y las políticas elegidas han influenciado la conformación de un contexto lesivo, favorable para la producción y reproducción de los accidentes de tránsito y, en particular, aquellos con motocicleta.

Almeida Filho (22-24) afirma que no hay impedimentos lógicos para la realización de estudios caso-control basados en agregados poblacionales, método aplicado en este análisis. Existen por otro lado limitaciones ajenas al diseño de un estudio ecológico debido al subregistro existente en los datos secundarios. Además, la categoría espacio está representada por los municipios y los datos utilizados reflejan el municipio como un todo, sin diferenciar sus singularidades intraespaciales. Otra limitación a considerar tiene que ver con el método de análisis cuantitativo (regresión logística multivariada) que verifica la asociación entre las variables desde una perspectiva de la causalidad lineal, además de no haber utilizado un análisis jerárquico. Sin embargo, se trató de minimizar esta cuestión utilizando un abordaje más amplio en la interpretación y reflexión de los resultados encontrados.

Motivos históricos y políticos producen el aumento de la razón del número de motocicletas por habitante en Brasil. La adquisición de una motocicleta está directamente asociada y favorecida por un modelo de industrialización, denominado "desarrollista", adoptado en Brasil y en el mundo globalizado, modelo que imprimió una lógica de mercado voraz orientado para la fabricación cada vez mayor de vehículos automotores veloces y prácticos como las motocicletas. La industria automovilística instalada en Brasil se volvió la columna vertebral de la industrialización en el país (25).
Según Vasconcelos (25), a partir de 1994 las políticas federales apoyaron la masificación del uso de motocicletas a través de leyes relativas a la producción, renuncia fiscal y facilidades de financiamiento. Entre 1992 y 2007, las ventas de motocicletas se multiplicaron por 12 veces, en tanto que las de automóviles lo hicieron por cuatro veces.

A partir de mediados de la década de 1990, la motocicleta pasó a ser intensamente empleada también como instrumento de trabajo, además de un medio de transporte; encajando perfectamente en los cambios derivados del comportamiento de la economía que privilegió el sector servicios e hizo crecer la informalidad, la precariedad el mercado de trabajo y el desempleo. El crecimiento del e-commerce, popularizado a través de Internet coincide también con esta época. Todo esto sucede bajo la égida de la desregulación tan apreciada en las relaciones de trabajo Ilamadas modernas, ampliándose así, un contingente de vulnerados.

Puede decirse que los trabajadores motociclistas son típicos representantes de las nuevas relaciones de trabajo capitalistas. Por lo tanto, en este orden, estos trabajadores que vinieron para quedarse se tornaron una pieza importante y con espacio en la intrincada malla socioeconómica de la sociedad contemporánea. Aunque se trate de una categoría estigmatizada, discriminada y condenada, es cada vez más solicitada.

Este nuevo actor en el tránsito, el motociclista, tiene que disputar el espacio con los demás existentes sin prácticamente ser reconocido, y lo ha hecho a la fuerza (25).

Ni la propia legislación vial resistió al poder de los fabricantes de motocicletas. Si bien el artículo 56 del Código de Tránsito Brasileño impedía la circulación de motocicletas entre filas de vehículos, fue vetado por la presidencia, con la justificación de que "Al prohibir al conductor de motocicletas y motonetas el pasaje entre vehículos de filas adyacentes, el dispositivo restringe de sobremanera la utilización de este tipo de vehículo, que en todo el mundo es ampliamente utilizado como forma de garantizar mayor agilidad de desplazamiento."

Con relación al factor de crecimiento de la población al año, característica de los municipios con mayor tasa de mortalidad por accidente de motocicleta; se sabe que el crecimiento acelerado 
y poco organizado provoca daños sociales, económicos y ambientales afectando incluso la salud de las personas (7). Sus consecuencias se hacen sentir también en el ambiente de circulación creando en los municipios áreas de conflicto cada vez mayores para los desplazamientos a pie o con vehículos no motorizados, además de un verdadero desarreglo urbano.

Los hallazgos de este estudio indican que las más bajas densidades demográficas presentan una mayor probabilidad de muerte por accidentes de motocicleta. Es un hecho que en esos lugares prácticamente no existe un sistema de transporte público colectivo urbano con ómnibus o similar. En Brasil, los ómnibus son gestionados por empresas privadas que buscan únicamente el lucro. Áreas con bajas densidades demográficas implican pocos pasajeros por quilómetro. Esto torna al servicio de transporte una operación poco lucrativa, obligando a que las personas opten por el uso de la motocicleta, mototaxis, taxis convencionales -lo que es mucho más caro- o por trasladarse a pie. El transporte y el movimiento de vehículos que antes eran de tracción animal fueron sustituidos abruptamente por la motocicleta, lo que ciertamente contribuyó a aumentar el número de accidentes.

En las ciudades con crecimiento desenfrenado de la flota total de vehículos, lo que no implica necesariamente áreas de mayor densidad poblacional como se observa en varios de los municipios analizados, se emplean técnicas de circulación que optimizan el desplazamiento solo de los automóviles, transformando el espacio urbano en peligroso para todos los que están en el tránsito, pero principalmente para aquellos cuyas características físicas y dinámicas son más vulnerables, tales como los peatones, ciclistas y motociclistas (26).

Por otro lado, en flagrante violación al Código de Tránsito, la municipalización del tránsito que podría ser un recurso para equilibrar esas disparidades no existe en la mayoría de los municipios. En el estado de Pernambuco, luego de más de 12 años de entrada en vigencia del código, hasta 2005 solo 18 municipios habían municipalizado el tránsito, número que en 2011 correspondía a 24 municipios.
Un componente de la dimensión económica, el PIB per cápita, indicó que cuanto más bajo, más probabilidades tiene el municipio de presentar mayores coeficientes de mortalidad por accidente de motocicleta, corroborando los resultados de otros estudios (27) que asocian la miseria y la pobreza con altas tasas de muertes por accidentes de tránsito, a pesar de que otros estudios encontraron resultados diferentes (21).

Vale resaltar que este artículo trata solamente de la mortalidad por accidentes de motocicleta, en tanto que existe otro aspecto tan grave o todavía más cruel que es la morbilidad derivada de estos accidentes. Por otro lado, se puede concluir también que los municipios que presentan el perfil aquí indicado, pueden estar señalando una deficiencia en la asistencia médica de urgencias.

Esta situación límite puede servir como un freno necesario, en el sentido de prevenir y reducir la violencia social tan exacerbada en los accidentes de motocicleta que, a su vez, es emblemática del contexto socioeconómico al que llegó la sociedad brasileña. Las respuestas podrán surgir del esfuerzo colectivo a través de una reevaluación de la actuación del Estado que busque el empoderamiento ecuánime de todos los actores involucrados, con acciones coherentes para la promoción de la seguridad y prevención de los accidentes.

\section{CONCLUSIONES}

Las variables relacionadas con la macropolítica (dimensión ecológico-política), que se mostraron más fuertemente asociadas con la mortalidad, son aquellas que constituyen el poder del Estado y cristalizan su esencia. Por lo tanto, con base en las evidencias de este estudio, se puede afirmar que los accidentes de tránsito, especialmente aquellos por motocicleta en Brasil, son emblemáticos del modelo de "desarrollo" elegido que considera apenas el crecimiento económico, ignorando los procesos de determinación y reproducción social, ciudadanía y derechos humanos. 


\section{AGRADECIMIENTOS}

Los autores agradecen a la Fundação de Amparo à Ciência e Tecnologia do Estado de Pernambuco (FACEPE) por el apoyo económico para el proyecto "Epidemiología de los accidentes de tránsito y análisis de las políticas públicas para la reducción de la morbimortalidad por accidente de tránsito con foco en los accidentes por motocicleta en el estado de Pernambuco", No. 076-221912437 (APQ 1341400/2008).

\section{REFERENCIAS BIBLIOGRÁFICAS}

1. Brasil, Ministério da Saúde, Departamento de Informática do Sistema Único de Saúde [Internet]. Sistema de informações sobre mortalidade. [citado 22 jul 2014] Disponible en http://www.datasus. gov.br.

2. Instituto de Pesquisa Econômica Aplicada, Associação Nacional de Transportes Públicos. Impactos sociais e econômicos dos acidentes de trânsito nas aglomerações urbanas: Relatório Executivo. Brasília: Denatran; 2003.

3. Instituto de Pesquisa Econômica Aplicada, Associação Nacional de Transportes Públicos. Impactos sociais e econômicos dos acidentes de trânsito nas rodovias brasileiras: Relatório Executivo. Brasília: Denatran; 2006.

4. Pavarino FRV. Morbimortalidade no trânsito: limitações dos processos educativos e contribuições do paradigma da promoção da saúde ao contexto brasileiro. Epidemiologia e Serviços Saúde. 2009;18 (4):375-384.

5. Naciones Unidas. Plan mundial para el decenio de acción para la seguridad vial 2011-2020 [Internet]. 2010 [citado 22 jul 2014]. Disponible en: http://goo.gl/Z7xVCe.

6. Minayo MCS. Seis características das mortes violentas no Brasil. Revista Brasileira de Estudos de População. 2009;26(1):135-140.

7. Morais-Neto OL, Montenegro MMS, Monteiro RA, Siqueira-Júnior JB, Silva MMA, Lima CM, Miranda LOM, Malta DC, Silva-Junior JB. Os autores respondem. Ciência \& Saúde Coletiva. 2012;17(9):2237-2245.

8. Silva PHNV, Lima MLC, Moreira RS, Souza WV, Cabral APS. Estudo espacial da mortalidade por acidentes de motocicleta em Pernambuco. Revista de Saúde Pública. 2011;45(2):409-415.

9. Samaja J. A reprodução social e a saúde: elementos metodológicos sobre a questão das relações entre saúde e condições de vida. Salvador: Casa da Qualidade Editora; 2000.
10. Samaja J. Epistemología de la salud: reproducción social, subjetividad y transdisciplina. Buenos Aires: Lugar Editorial; 2007.

11. Santos M. A natureza do espaço: técnica e tempo, razão e emoção. São Paulo: Hucitec; 1996.

12. Samaja J. Desafios a la epidemiologia (pasos para uma epidemiologia Miltoniana). Revista Brasileira de Epidemiologia. 2003;6(2):105-120.

13. Almeida Filho N. Epidemiologia sem números. Rio de Janeiro: Campus; 1989.

14. Almeida Filho N, Rouquayrol MZ. Elementos de metodologia epidemiológica. En: Rouquayrol MZ, Almeida Filho N. Epidemiologia e Saúde. Rio de Janeiro: Medsi; 2003.

15. Assunção RM, Barreto SM, Guerra HL, Sakurai E. Figuras de taxas epidemiológicas: uma abordagem bayesiana. Cadernos de Saúde Pública. 1998;14:713-723.

16. Bailey TC, Gatrel AC. Interactive spatial data analysis. Essex: Longman Scientific \& Technical; 1995.

17. Bayerl E. Contribuição à análise dos acidentes de trânsito a partir da evolução da taxa de motorização e indicadores socioeconômicos no Brasil [Dissertação de Mestrado]. Rio de Janeiro: Universidade Federal do Rio de Janeiro; 2006.

18. Campos MM. Uma análise da relação entre acidentes de tráfego e variáveis sociais, econômicas, urbanas e mobilidade na cidade do Rio de Janeiro [Dissertação de Mestrado]. Rio de Janeiro: Universidade Federal do Rio de Janeiro; 2005.

19. Macías GR. A complexidade da situação epidemiológica dos acidentes de trânsito, 2009. [Tese de Doutorado]. Salvador: Universidad Federal da Bahia; 2009.

20. Macías GR, Almeida Filho N, Alazraqui M. Análisis de las muertes por accidentes de tránsito en el municipio de Lanús, Argentina, 1998-2004. Salud Colectiva. 2010;6(3):313-328. 
21. Souza ER, Minayo MCS, Malaquias JV. Violência no trânsito-expressão da violência social. En: Brasil, Ministério da Saúde. Impacto da violência na saúde dos brasileiros. Brasília: MS; 2005.

22. Almeida Filho N. Transdisciplinaridade e o paradigma pós-disciplinar na saúde. Saúde e Sociedade. 2005;14(3):30-50.

23. Almeida Filho N, Coutinho D. Causalidade, contingência, complexidade: o futuro do conceito de risco. Physis. 2007;17(1):95-137.

24. Almeida Filho N. A Saúde e o paradigma da complexidade. Cadernos IHU. 2006;4(15):1-47.
25. Vasconcelos EA. O custo social da motocicleta no Brasil. Revista dos Transportes Públicos. 2008;119(20):127-142.

26. Pavarino FRV. Aspectos da educação de trânsito decorrentes das proposições das teorias da segurança, problemas e alternativas. Transportes. 2004;12(1):59-68.

27. Trindade J, Nassi D. A miséria como causa dos acidentes de trânsito no Brasil [Internet]. s/f [citado 22 jul 2014]. Disponible en: http://www.sinaldetransito.com.br/artigos/miseria.pdf.

FORMA DE CITAR

Silva PHNV, Lima MLC, Souza WV, Moreira RS, Oliveira FJM. Muertes por accidente de motocicleta y su asociación con variables relacionadas a la reproducción social en un estado del noreste brasileño. Salud Colectiva. 2015;11(3):401-410.

Recibido: 14 de octubre de 2014 | Versión final: 24 de marzo de 2015 | Aprobado: 19 de abril de 2015

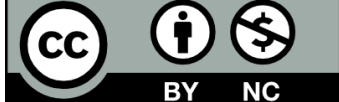

Este obra está bajo una licencia de Creative Commons Reconocimiento-NoComercial 4.0 Internacional. Reconocimiento - Permite copiar, distribuir y comunicar públicamente la obra. A cambio, se debe reconocer y citar al autor original. No Comercial - Esta obra no puede ser utilizada con finalidades comerciales, a menos que se obtenga el permiso.

http://dx.doi.org/10.18294/sc.2015.724 